ARTICLE

\title{
Ultra-fast single-crystal polymerization of large-sized covalent organic frameworks
}

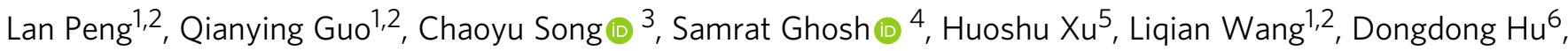 \\ Lei Shi (iD ${ }^{3}$, Ling Zhao ${ }^{6}$, Qiaowei $\mathrm{Li}^{5}$, Tsuneaki Sakurai ${ }^{4}$, Hugen Yan (iD ${ }^{3}$, Shu Seki ${ }^{4}$, Yunqi Liu ${ }^{2,7} \&$ \\ Dacheng Wei (iD $1,2 \times$
}

In principle, polymerization tends to produce amorphous or poorly crystalline materials. Efficiently producing high-quality single crystals by polymerization in solvent remains as an unsolved issue in chemistry, especially for covalent organic frameworks (COFs) with highly complex structures. To produce $\mu \mathrm{m}$-sized single crystals, the growth time is prolonged to $>15$ days, far away from the requirements in practical applications. Here, we find supercritical $\mathrm{CO}_{2}\left(\mathrm{sc}-\mathrm{CO}_{2}\right)$ accelerates single-crystal polymerization by $10,000,000$ folds, and produces two-dimensional (2D) COF single crystals with size up to $0.2 \mathrm{~mm}$ within $2 \sim 5 \mathrm{~min}$. Although it is the fastest single-crystal polymerization, the growth in $\mathrm{sc}-\mathrm{CO}_{2}$ leads to not only the largest crystal size of 2D COFs, but also higher quality with improved photoconductivity performance. This work overcomes traditional concept on low efficiency of single-crystal polymerization, and holds great promise for future applications owing to its efficiency, industrial compatibility, environmental friendliness and universality for different crystalline structures and linkage bonds.

\footnotetext{
${ }^{1}$ State Key Laboratory of Molecular Engineering of Polymers, Department of Macromolecular Science, Fudan University, Shanghai, China. ${ }^{2}$ Institute of Molecular Materials and Devices, Fudan University, Shanghai, China. ${ }^{3}$ Department of Physics, Fudan University, Shanghai, China. ${ }^{4}$ Department of Molecular Engineering, Graduate School of Engineering, Kyoto University, Nishikyo-ku, Kyoto, Japan. ${ }^{5}$ Department of Chemistry, Fudan University, Shanghai, China. ${ }^{6}$ School of Chemical Engineering, East China University of Science and Technology, Shanghai, China. ${ }^{7}$ Institute of Chemistry, Chinese Academy of Sciences,

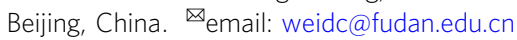


$\mathrm{t}$ is generally considered that the growth of a single crystal is much more time-consuming than amorphous or polycrystalline materials ${ }^{1}$. Compared with other crystals, crystalline materials constructed via covalent bonds face a greater challenge in efficient and precise production, since the formation and breakage of strong covalent bonds have lower reversibility than other linkages ${ }^{2-6}$. Single-crystal growth normally requires harsh conditions such as high temperatures or pressures ${ }^{7}$. Under a mild condition of polymerization in solvent, the challenge becomes more $\mathrm{critical}^{2,4,5,7}$. To obtain high-quality single crystals, it is necessary to decelerate the polymerization and prolong the growth time ${ }^{8,9}$. Considerable longtime is consumed for the polymerization along with reversible errorcorrection of the covalent lattices $4,6,7,10-13$. Therefore, although covalent bond ensures higher stability of crystalline materials in the application, it brings single-crystal polymerization a puzzling contradiction between growth time and product quality, causing dilemma in not only researches but also applications of these materials $2,7,10,11$. As a type of crystalline porous polymers, covalent organic frameworks (COFs) are obtained by atomically precise integration of organic building blocks via strong covalent bonds in a highly ordered periodic manner ${ }^{10,11,14-16}$. They have highly complex structures with different topologies, pore structures, linkages, functional groups, etc. and the interaction strength between intraand inter-layers of two-dimensional (2D) COFs differ by several orders of magnitude $e^{3,10,11,14}$. Owing to special well-defined stable structures together with tailored functionalities, they have attracted numerous interests since 2005 and promised widespread applications ranging from gas separation to photoelectronics ${ }^{10,11,15-20}$. However, such a highly complex structure, together with the fundamental limitation from single-crystal polymerization, gives rise to a great difficulty in the fast growth of high-quality samples, especially large-sized single crystals.

By solvothermal, ionothermal, mechanochemical, or microwave polymerization, only polycrystalline powders comprising disorderly aggregated crystallites smaller than $200 \mathrm{~nm}$ are obtained, even when the whole reaction lasts for $1-4$ days $^{15,17-22}$, hampering state-ofthe-art applications of single-crystalline COFs. Although numerous researchers attempt to accelerate the polymerization, the sample quality is normally poor with small grain sizes $5,23-25$. To obtain 1-2 $\mu \mathrm{m}$-sized single crystals, it is required to decelerate the polymerization by decreasing the addition rate of monomers 8,26 , and the time is prolonged to 15 days or longer. In industrial-scale production, long reaction time means high cost, low efficiency, and poor feasibility. Therefore, existing knowledge and technologies are still far away from the requirement of practical applications in efficiently and precisely producing covalent single crystals by polymerization. More understanding on single-crystal polymerization is required. Herein, we demonstrate the first example of ultrafast single-crystal polymerization in solvent, and find the pivotal role of solvent medium in the process. Compared with traditional liquids, sc- $\mathrm{CO}_{2}$ extremely accelerates nucleation, polymerization as well as the reversible error-checking and proof-reading. We develop a methodology, named supercritically solvothermal polymerization, and realize ultra-fast growth of high-yield COF single crystals with different crystalline structures and linkage bonds. The crystal size is up to $0.2 \mathrm{~mm}, \sim 100$ times larger than $2 \mathrm{D}$ COF single crystals reported previously ${ }^{8}$. The growth rate reaches $40 \mu \mathrm{m} \mathrm{min}^{-1}$, up to 100,000 times faster than the best results of growing 2D COF single crystals $^{8,9}$ and around 6000 times faster than ultra-fast polymerization technologies of producing polycrystalline or nanocrystalline $\mathrm{COFs}^{23-25}$

\section{Results}

Single-crystal polymerization in sc- $\mathrm{CO}_{2}$. Three types of $2 \mathrm{D}$ COFs (sc-COFs, Fig. 1, Supplementary Fig. 1) with different structures and linkage bonds are prepared in $\mathrm{sc}-\mathrm{CO}_{2}\left(80^{\circ} \mathrm{C}\right.$, $8 \mathrm{MPa}$ ) with $0.25 \%$ (vol.) n-butyl alcohol ( $\mathrm{n}-\mathrm{BuOH})$, including imine-linked $\mathrm{COFs}\left(\mathrm{COF}_{\mathrm{TP}-\mathrm{Py}}, \mathrm{COF}_{\mathrm{TB}-\mathrm{BA}}\right)$ and boronate esterlinked COFs $\left(\mathrm{COF}_{5}\right)$. These COFs are also synthesized by solvothermal polymerization (os-COFs) in $50 \%$ (vol.) $\mathrm{n}-\mathrm{BuOH}$ and $50 \%$ (vol.) 1,2-dichlorobenzene (o-DCB) as a comparison. After 5 min growth in $\mathrm{sc}-\mathrm{CO}_{2}$, Fourier transform infrared (FT-IR) spectra (Supplementary Fig. 2) confirm the polymerization. Optical microscope (OM) and scanning electron microscope (SEM) images (Figs. 1c, d, Supplementary Figs. 3-5) images reveal that all of the as-grown sc-COFs have high-yield rod-like morphologies with a length above $\mu \mathrm{m}$-scale, distinct from that of disorderedly aggregated os-COFs (3 days). In particular, most sc$\mathrm{COF}_{\mathrm{TP}-\mathrm{Py}}(5 \mathrm{~min})$ are $10-40 \mu \mathrm{m}$ in length (Supplementary Fig. 6). We also grow sc-COF ${ }_{\mathrm{TP}-\mathrm{Py}}$ for $2 \mathrm{~min}$, and the length reaches up to $30 \mu \mathrm{m}$ (Supplementary Fig. 7). Powder X-ray diffraction (PXRD) patterns (Fig. 1e) of the sc-COFs present sharp diffraction peaks with the highest in small-angle region, indicating that all sc-COFs (5 min or $90 \mathrm{~min}$ ) are highly crystalline. The peak positions are in good agreement with the simulated patterns of AA eclipsed stacking models, which match well with previous literatures ${ }^{15,27,28}$ and PXRD of corresponding os-COFs (3 days). No diffraction peaks appear in PXRD of os-COFs (30 min) or no samples (os$\mathrm{COF}_{5}, 90 \mathrm{~min}$ ) are obtained, indicating that the crystallization in organic solvent is much slower than that in $\mathrm{sc}-\mathrm{CO}_{2}$.

Crystalline structure. Transmission electron microscope (TEM) images and selected area electron diffraction (SAED) patterns (Fig. 2a-j, Supplementary Figs. 8, 9, 10) reveal high yield and highly crystalline features of the rod-like sc-COFs. The lattice fringes are clearly observed along the length direction with an inter-lattice separation of $2.0 \mathrm{~nm}\left(\mathrm{sc}-\mathrm{COF}_{\mathrm{TB}-\mathrm{BA}}\right), 2.4 \mathrm{~nm}\left(\mathrm{sc}-\mathrm{COF}_{5}\right), 1.8$ or $2.3 \mathrm{~nm}$ (sc-COF $\mathrm{CP}_{\mathrm{TP}-\mathrm{Py}}$ ), which correspond to $\mathrm{COF}_{\mathrm{TB}-\mathrm{BA}}(100), \mathrm{COF}_{5}(100)$, $\mathrm{COF}_{\mathrm{TP}-\mathrm{Py}}(020)$ or $\mathrm{COF}_{\mathrm{TP}-\mathrm{Py}}(110)$ planes, respectively, indicating the 2D COF layers are stacked along the length direction via [001]. Cross-section TEM images (Fig. 2b) show clear fringes of sc-COF $\mathrm{CB}_{\mathrm{TB}}$ BA with corresponding six-fold-symmetric fast Fourier transform (FFT) patterns, thus the micro-pores of $2 \mathrm{D}$ sc-COFs are oriented parallel to the rods. Although some rods are curved owing to the flexibility, all locations of the rod are well crystalline, and the microporous channels with open ends are continuous over the entire rod region without domain boundaries or interfaces (Fig. 2g-j, Supplementary Figs. 11, 12). The same set of SAED patterns are collected from different locations, indicating the single-crystalline nature of the $2 \mathrm{D}$ sc-COF rods (Supplementary Fig. 13). The sc$\mathrm{COF}_{\text {TP-Py }}$ grown for $2 \mathrm{~min}$ also has the same feature with highquality single-crystalline structure (Supplementary Fig. 14). In contrast, all os-COFs are disorderedly polycrystalline with nm-scale domains (Fig. 2k, 1, Supplementary Fig. 15). Cross-polarized OM images (Fig. 2m, n, Supplementary Fig. 16) confirm single crystallinity of the sc-COFs, as uniform polarized light extinction is observed over the entire length of the rods (Supplementary Video 1), different from os-COFs (Supplementary Fig. 17, Supplementary Video 2). Some sc- $\mathrm{COF}_{\mathrm{TP}-\mathrm{Py}}$ crystals are larger than $0.1 \mathrm{~mm}$, up to $0.20 \mathrm{~mm}$, which are the largest 2D COF single crystals reported till now (Fig. 1b).

Growth rate and crystal quality. To study the growth rate, $2 \mathrm{D}$ COFs are grown for different times. SEM images (Supplementary Figs. 7,18 ) show rod-like crystals with sizes up to several tens (sc$\mathrm{COF}_{\mathrm{TP}-\mathrm{Py}}$ ) of micrometers are obtained within 2-5 min, with similar morphologies and lengths to sc-COFs grown for $2 \mathrm{~h}$ and $12 \mathrm{~h}$ (Supplementary Fig. 19), indicating that most single-crystal polymerization finishes within $5 \mathrm{~min}$ owing to the consuming monomer. The os-COFs have distinct morphologies (Supplementary Fig. 20), 
a

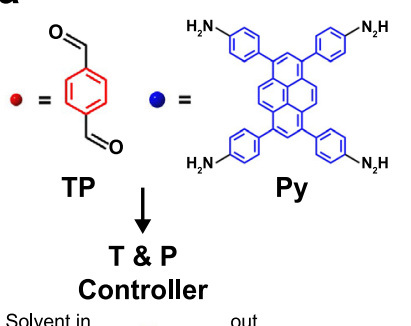

Product:
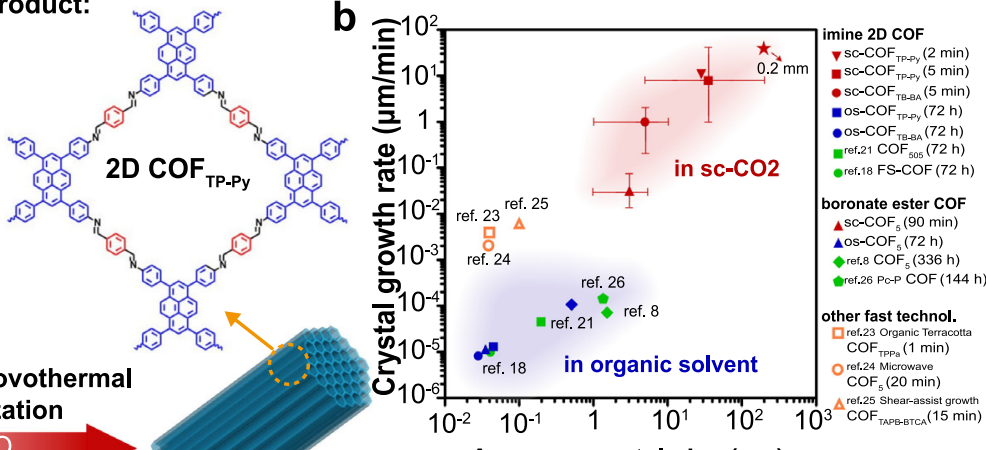

Average crystal size $(\mu \mathrm{m})$

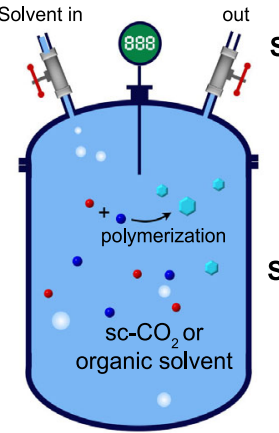

Supercritical-sovothermal polymerization
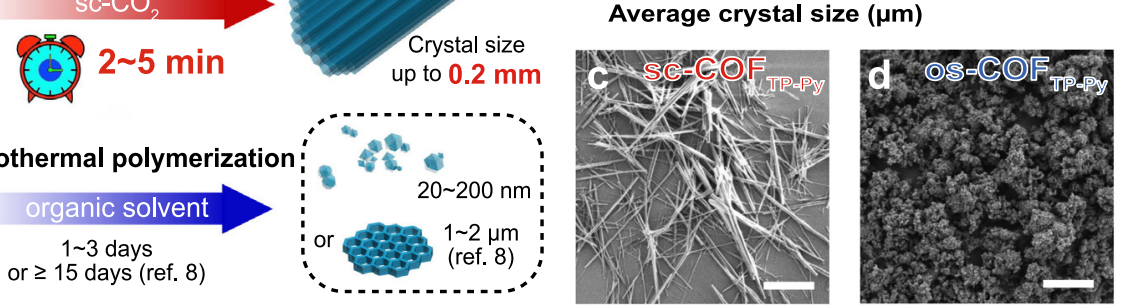

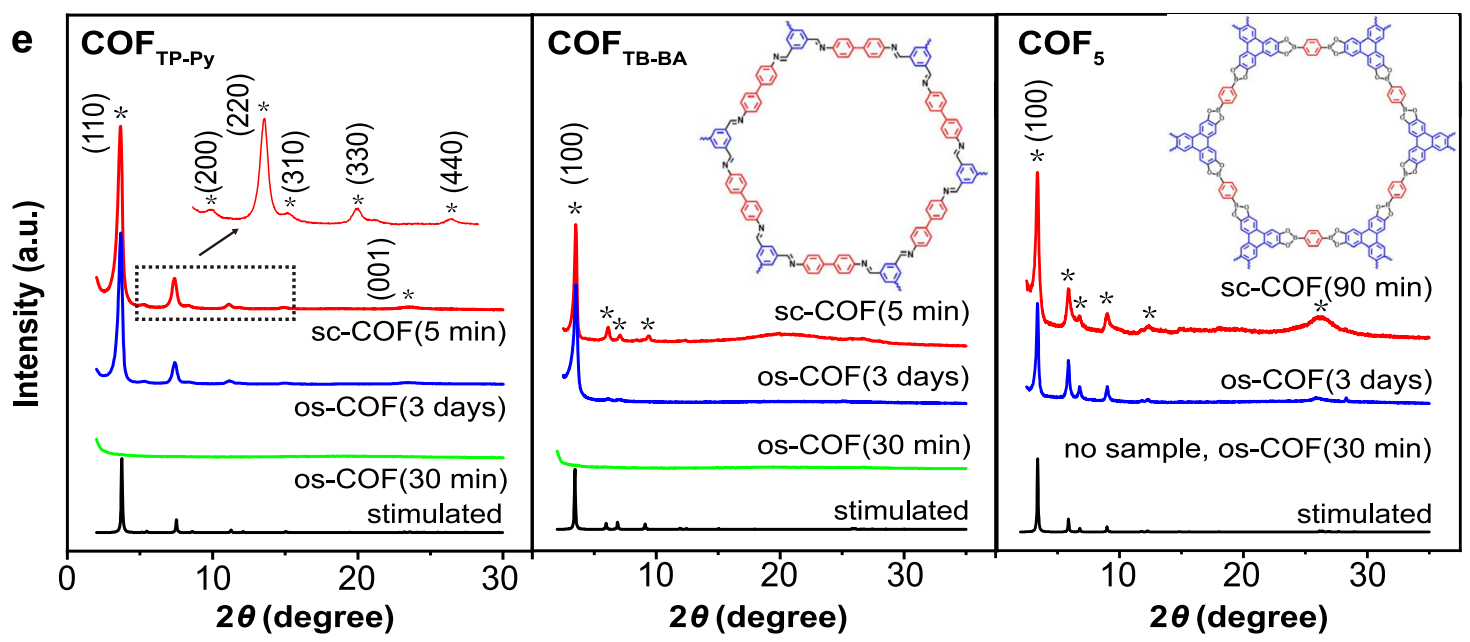

Fig. 1 Supercritically solvothermal polymerization. a Schematic of the COFTP-Py synthesis. b Crystal size and growth rate in this work (sc-COFs and os-COFs) compared with some best-reported results. The red star indicates the best result in Sc-CO $2 . \mathbf{c}, \mathbf{d}$ SEM images of sc-COF PXRD patterns of sc-COFs ( 5 or 90 min), os-COFs (3 days), os-COFs (30 or 90 min), and the simulated patterns. The characteristic peaks are marked by stars. The scale bars are $10 \mu \mathrm{m}$.

which are disorderedly aggregated with small crystallites, even if the growth lasts for 3 days. The crystal growth rate of sc-COFs reaches $40 \mu \mathrm{m} \mathrm{min}^{-1}$ in sc- $\mathrm{CO}_{2}$, up to 7 orders of magnitude faster compared with that of os-COFs (Fig. 1b).

The crystal quality is measured by PXRD. High-intensity diffraction peaks of sc-COF $\mathrm{TP}_{\mathrm{TP}-\mathrm{Py}}$ appear after 2-5 min (Fig. 3a), which are similar to PXRD patterns of sc- $\mathrm{COF}_{\mathrm{TP}-\mathrm{Py}}$ grown for $8-12 \mathrm{~h}$ and os- $\mathrm{COF}_{\mathrm{TP}-\mathrm{Py}}$ grown for 3 days, indicating high-quality $\mathrm{COF}$ are obtained in $\mathrm{Sc}-\mathrm{CO}_{2}$ within minutes. Weak diffraction peaks of the solvothermal samples appear only after $2 \mathrm{~h}$, indicating the crystallization in organic solvent is much slower. The full width at half maximum (FWHM) of the diffraction peak (Fig. 3b) is related to the crystal quality ${ }^{29}$. In $\mathrm{sc}-\mathrm{CO}_{2}, \mathrm{FWHM}$ of the $\mathrm{sc}-\mathrm{COF}_{\mathrm{TP}-\mathrm{Py}}(110)$ peak reaches $0.39^{\circ}$ after $5 \mathrm{~min}$, decreases slightly to $0.35^{\circ}$ after $2 \mathrm{~h}$, and maintains around $0.34^{\circ}$ until $8 \mathrm{~h}$. However, in organic solvent, FWHM of the os-COF $\mathrm{CO}_{\mathrm{TP}-\mathrm{Py}}$ (110) peak is only $0.62^{\circ}$ after $4 \mathrm{~h}$. A time up to 1-3 days is required to obtain high-quality COFs with FWHM around $0.35^{\circ}$. Moreover, we use a mixture solvent of sc- $\mathrm{CO}_{2}$ and $\mathrm{n}-\mathrm{BuOH}$ (Fig. $3 \mathrm{c}$ ). Higher sc- $\mathrm{CO}_{2}$ content leads to a larger growth rate and smaller FWHM of $\mathrm{COF}_{\mathrm{TP}-\mathrm{Py}}(110)$ peak, indicating that the solvent medium plays a pivotal role in not only ultra-fast crystallization but also the formation of high-quality crystalline structures.

Single-crystal polymerization mechanism. Ultra-fast singlecrystal polymerization is related to the special properties of sc$\mathrm{CO}_{2}$. In organic solvent, the COF crystals are obtained by initial polymerization and nucleation followed by a subsequent crystalline growth or transformation process $3,5,6,11-13$. Similarly, the growth of $2 \mathrm{D}$ COFs in sc- $\mathrm{CO}_{2}$ involves extension of the lattice in the lateral [100] direction by covalent bond formation as well as in the vertical [001] direction by non-covalent stacking (Fig. 4) ${ }^{12,13}$. The assembly of monomers into the ordered framework by polymerization normally encounters many mistakes, such as overlays, defect sites, ill-defined agglomerations, interlayer cross-links, etc ${ }^{4}$. To obtain an ordered framework, a time-consuming reversible selfcorrection is required along with the polymerization for error-checking and proof-reading $4,6-13$. Considering the large surface tension $\left(20-50 \mathrm{dyn}^{-1}\right)$ and viscosity $(0.3-4 \mathrm{cp}$, normally) of organic solvent, the transport of precursors 


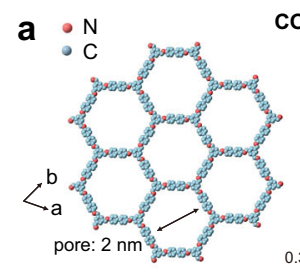

Top view
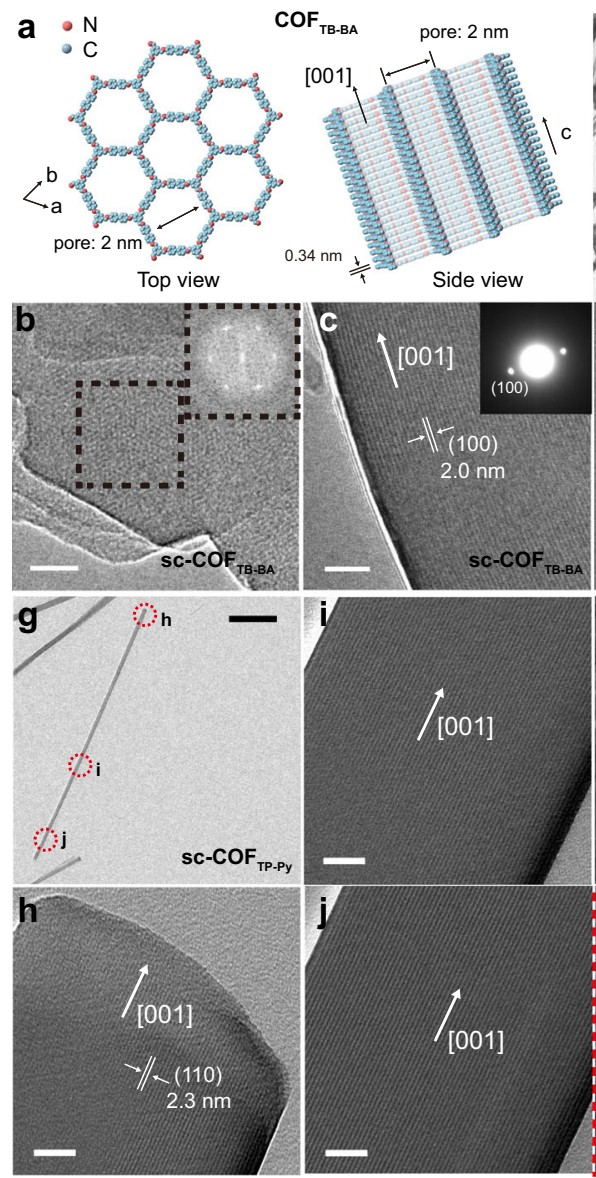
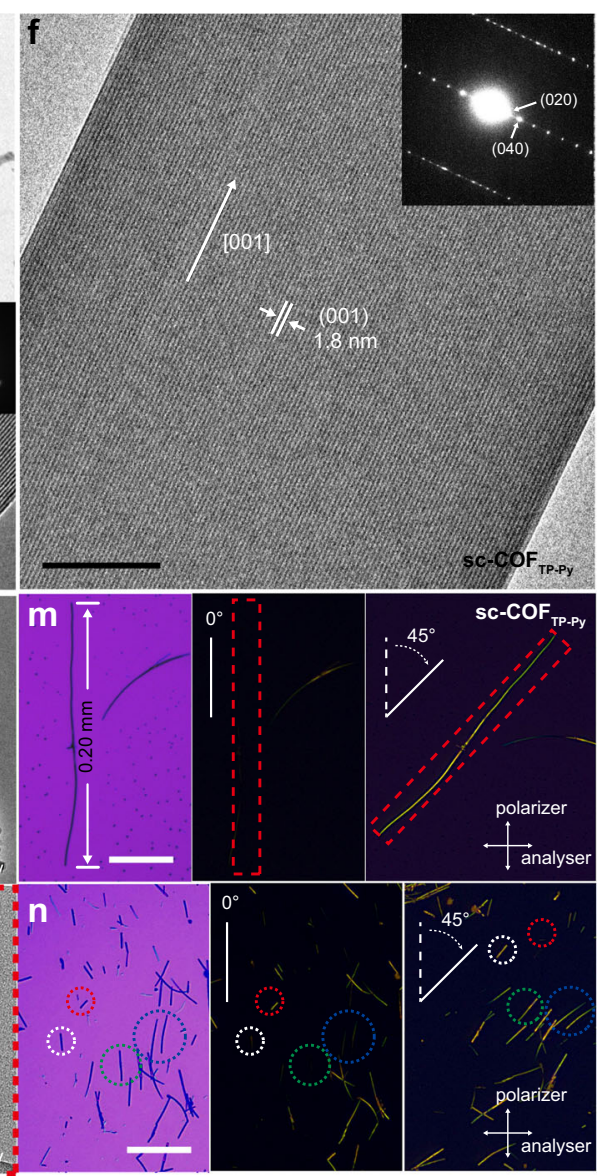

Fig. 2 Characterization of the crystalline structure. a Top and side view of COF $\mathrm{TB}_{\mathrm{BA}}$. b Cross-section TEM image of sC-COF $\mathrm{TB}_{\mathrm{BA}}$. The inset is the FFT

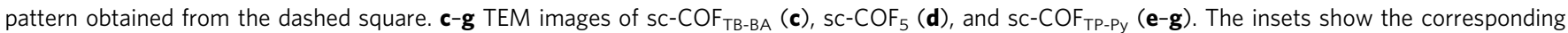

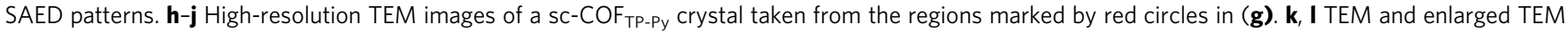
images of os-COF TP-Py. $_{\text {m, }} \mathbf{n}$ OM and cross-polarized OM images of a 0.20 mm long sc-COF are $20 \mathrm{~nm}$ in (b-d), (h-j), $50 \mathrm{~nm}$ in (f, I), $200 \mathrm{~nm}$ in (k), $2 \mu \mathrm{m}$ in $(\mathbf{g}), 10 \mu \mathrm{m}$ in (e), $50 \mu \mathrm{m}$ in (m) and (n).
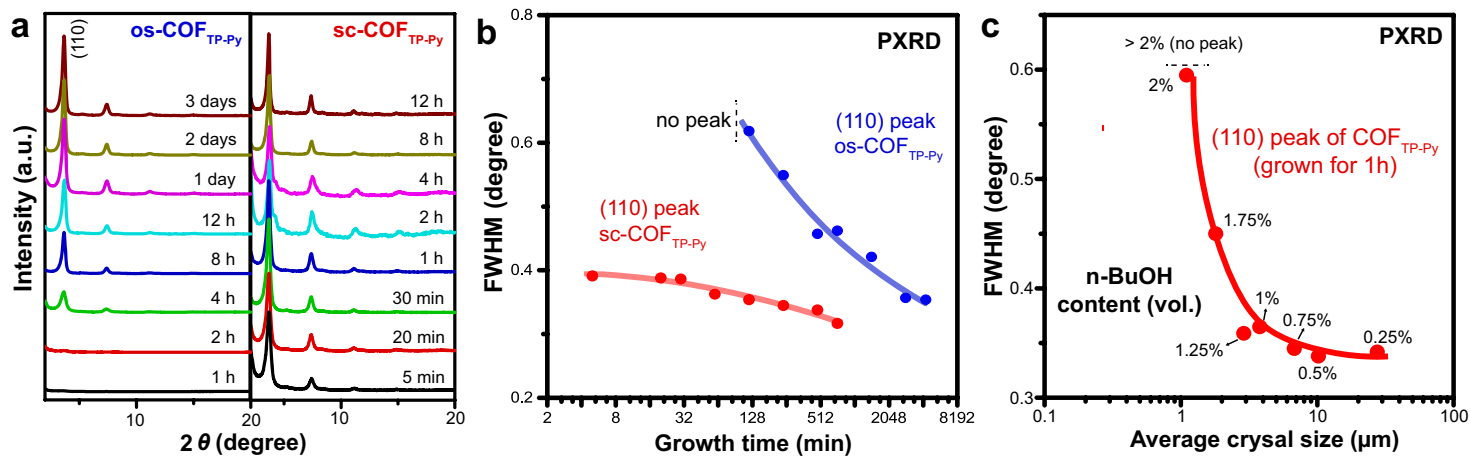

Fig. 3 Ultra-fast crystal growth of sc-COFs. a PXRD of the samples of os-COFTP-Py and Sc-COF ${ }_{T P-P y}$ grown for different times. $\mathbf{b}$ FWHM of Sc-COFTP-Py

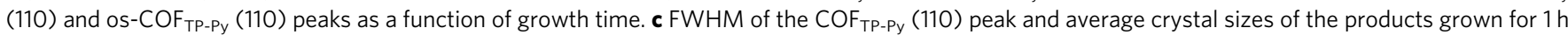
in a mixture solvent of $\mathrm{n}-\mathrm{BuOH}$ and $\mathrm{sc}-\mathrm{CO}_{2}$.

(including monomers and oligomers) and byproducts to or from the reaction sites is restricted by the slow diffusion rate in the solvent as well as in the micro-pores or interstices of COFs. As a result, the precursor concentration is low while the byproduct concentration is high near the reaction sites, which drastically slows down the reversible reaction, decelerates the framework formation, and brings a fundamental limitation in ultra-fast polymerization of high-quality single crystals.
Supercritical fluid technologies have been generally utilized in pharmaceutical, food, chemical manufacturing, and industries since $1960 \mathrm{~s}^{30}$, which realize a green, scalable and efficient chemical or biochemical synthesis and processing in a special medium distinct from normal gases and liquids ${ }^{31-33}$. Considering the inertness, sc- $\mathrm{CO}_{2}$ functions as an inert solvent in the reactions ${ }^{34}$, and the reaction thermodynamics is similar to that in liquid mediums. The difference is that the supercritical fluid 


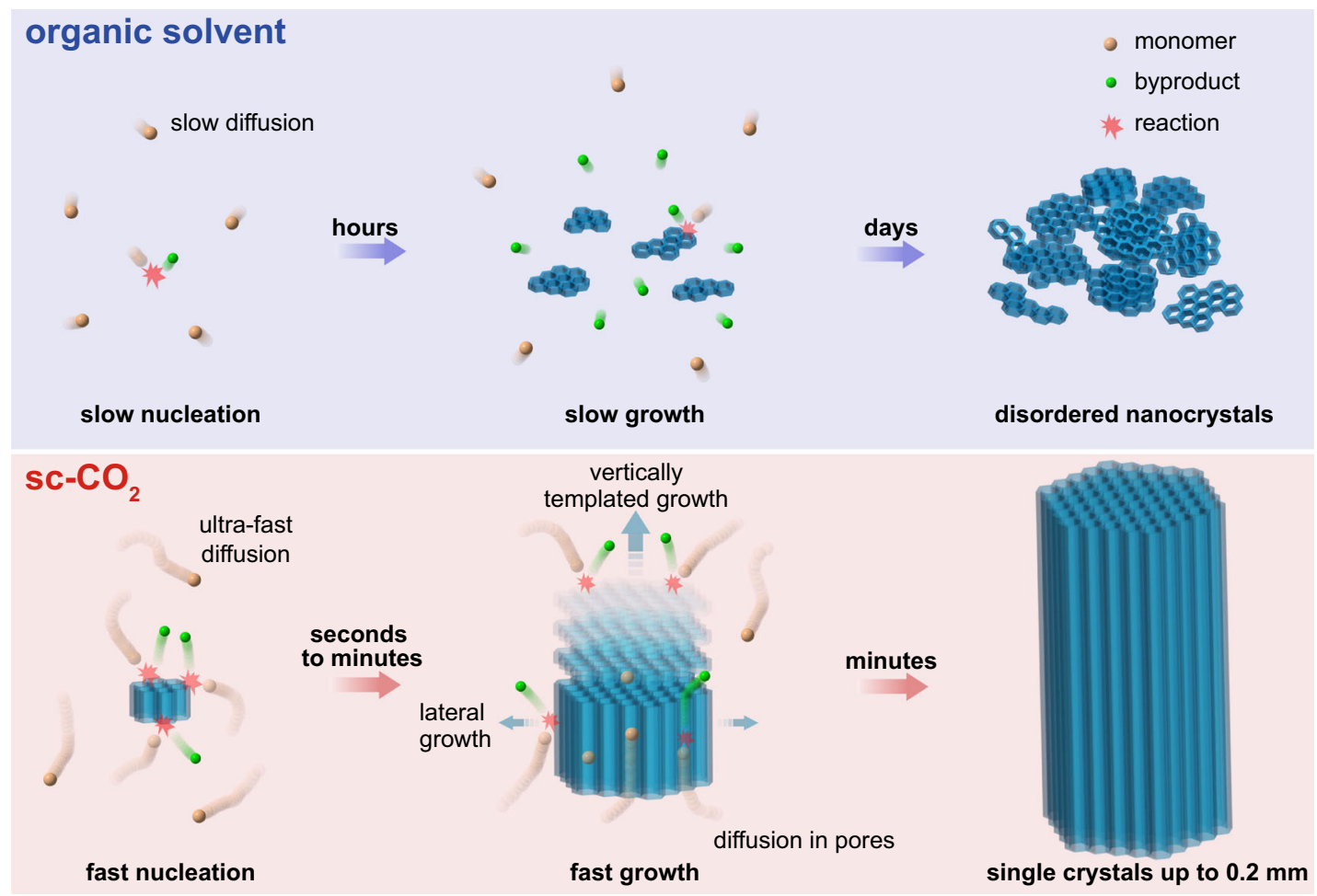

Fig. 4 Single-crystal polymerization mechanism. Schematic illustration of polymerization in organic solvent or sc- $\mathrm{CO}_{2}$. In sc- $\mathrm{CO}_{2}$, the ultra-fast diffusion of monomers, oligomers, and byproducts in medium and micro-pores accelerates polymerization as well as error-checking and proof-reading, while the suitable solubility accelerates vertically epitaxial growth, resulting in minutes-growth of single crystals.
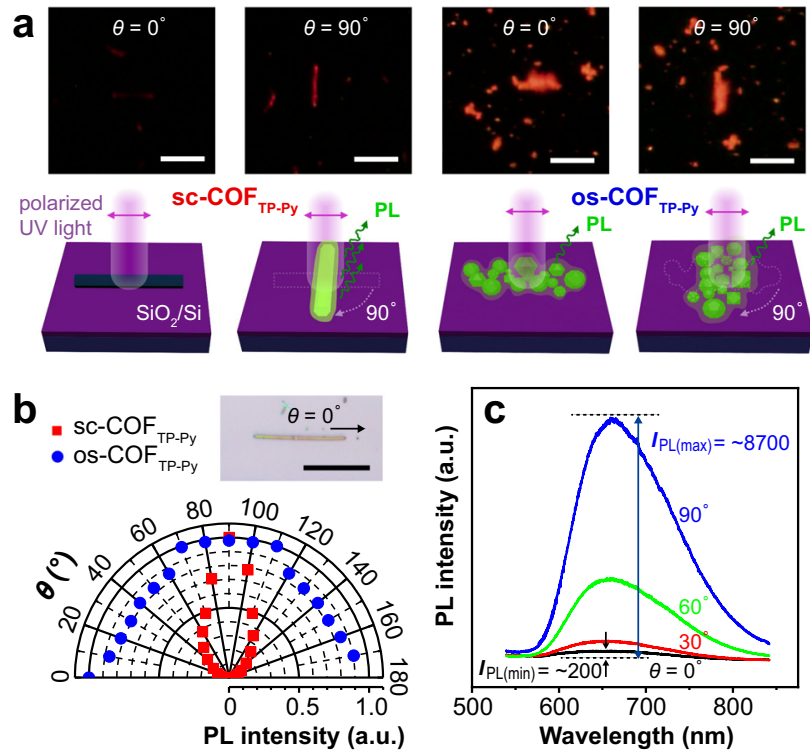

Fig. 5 Polarized photoluminescence. a Polarized fluorescence microscopy images and schematic illustration of Sc-COF $\mathrm{CP}_{\mathrm{TPy}}$ and os-COF $\mathrm{CP}_{\mathrm{TPy}}$ at $\theta=0^{\circ}$ and $90^{\circ}$. b Angle-dependent PL intensity at different $\theta$. The inset is an

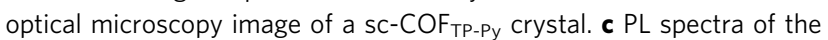
crystal when $\theta$ is $0^{\circ}, 30^{\circ}, 60^{\circ}, 90^{\circ}$. The DR (defined as $I_{\mathrm{PL}(\max )} / I_{\mathrm{PL}(\min )}$ ) is calculated to be 43.5 . The scale bars are $10 \mu \mathrm{m}$ in (a), $20 \mu \mathrm{m}$ in (b).

combines gas-like viscosity and surface tension with liquid-like density and solvating properties ${ }^{35}$. The viscosity of $\mathrm{sc}-\mathrm{CO}_{2}$ is about $0.02 \mathrm{cp}\left(80^{\circ} \mathrm{C}, 8 \mathrm{MPa}\right)$, while the surface tension is near zero $^{36}$. The diffusion coefficient is $1-2$ orders of magnitude higher than that in organic solvent ${ }^{37}$. As a result, sc- $\mathrm{CO}_{2}$ allows increased penetrating ability and much faster diffusion of precursors and by-products in the medium and in the micropores or interstices of $\mathrm{COFs}^{38}$. It is expected that the precursors can be sufficiently supplied to the reaction sites while the byproducts be fast removed from there. Thus, $\mathrm{sc}-\mathrm{CO}_{2}$ greatly influences the reaction kinetics and accelerates the polymerization along with the error-checking and proof-reading process, resulting in ultra-fast nucleation and extension of lateral covalent frameworks. As an evidence, we grow 2D COFs in the nanometer-scale interstices of highly ordered 3D monolith made of $1 \mu \mathrm{m}$-sized polystyrene spheres (PSs). After removing PSs, a free-standing $3 \mathrm{D}$-ordered structure of sc-COFs is obtained (Supplementary Figs. 21, 22). As a comparison, in 1,4-dioxane and mesitylene mixture, no COFs can grow in the interstices of the 3D monolith (Supplementary Fig. 23). This result indicates high diffusion and penetrating ability in $\mathrm{sc}-\mathrm{CO}_{2}$. To obtain an eclipsed non-covalent stacking structure of 2D COFs, a new layer forms via oligomer stacking and subsequent lateral template polymerization on top of an old layer ${ }^{13}$. The solubility of supercritical fluid is intermediate between that of a gas and a liquid ${ }^{33}$. The moderate solubility of sc- $\mathrm{CO}_{2}$ allows nucleation and efficient stacking of oligomers vertically on old layers (Supplementary Fig. 24), resulting in the growth of the crystal along [001] direction.

To prove the mechanism, control experiments tune solvent property by adding $\mathrm{n}-\mathrm{BuOH}$ in $\mathrm{sc}-\mathrm{CO}_{2}$. With higher $\mathrm{n}-\mathrm{BuOH}$ content, the mixture solvent has lower diffusion ability and larger solubility. As a result, the products $(1 \mathrm{~h})$ have reduced crystal length (Supplementary Figs. 25-27) and increasing FWHM of $\mathrm{COF}_{\mathrm{TP}-\mathrm{Py}}(110)$ peak (Fig. $3 \mathrm{c}$ ), when $\mathrm{n}-\mathrm{BuOH}$ content increases from $0.25 \%$ (vol.) to $2 \%$ (vol.). Finally, the products become amorphous without any diffraction peaks when the content is $>2 \%$ (vol.). This result indicates that the solvent medium plays a pivotal role in not only ultra-fast growth but also the formation of 
high-quality crystalline structures. Therefore, fast revisable reaction as well as efficient epitaxial nucleation and growth of the new layers in $\mathrm{sc}-\mathrm{CO}_{2}$ realize minutes-growth of $\mathrm{COFs}$ into large-sized rod-like single crystals.

Polarized photoluminescence. We measure the photoluminescence (PL) emission from sc- $\mathrm{COF}_{\mathrm{TP}-\mathrm{Py}}$ and os- $\mathrm{COF}_{\mathrm{TP}-\mathrm{Py}}$ (Fig. 5a). Angle-dependent emission only occurs in the case of sc$\mathrm{COF}_{\mathrm{TP}-\mathrm{Py}}(5 \mathrm{~min})$. When the polarization angle $(\theta)$ changes from $0^{\circ}$ to $180^{\circ}$ (the long axis direction is set to $0^{\circ}$ ), the PL emission $\left(I_{\mathrm{PL}}\right)$ from the crystal (Fig. 5b) gradually becomes stronger $\left(0^{\circ}-90^{\circ}\right)$, and then becomes weaker $\left(90^{\circ}-180^{\circ}\right)$. The dichroic ratio (DR) (defined as $I_{\mathrm{PL}}$ maximum $/ I_{\mathrm{PL}}$ minimum) is as high as 43.5 , and the polarization ratio $(\rho)^{39}$, defined as $(\mathrm{DR}-1) /(\mathrm{DR}+1)$, is 0.96 (Fig. 5c). In the case of os- $\mathrm{COF}_{\text {TP-Py }}$ (3 days) or COFs produced by other methods, the $\rho$ is only $\sim 0$ (Fig. 5b). It is the first report of polarized PL emission from COFs. The $\rho$ is among the highest values of organic or polymeric crystalline materials ${ }^{40}$, which verifies not only single-crystalline nature but also ultrahigh crystalline quality of sc-COF $\mathrm{CP}_{\mathrm{TP}-\mathrm{Py}}$ (see Supplementary Note 1$)^{41}$.

FP-TRMC photoconductivity. In our measurement, the spatial size of the oscillating motion of charge carriers is estimated within several nanometers at a maximum ${ }^{42}$. Thus, the grain and/ or domain boundary will not affect the $\phi \Sigma \mu$ obtained by FPTRMC. The FP-TRMC result is not related to the grain size but the actual quality (or crystallinity) of the as-grown $\mathrm{COF}^{43}$.

We measure the samples by flash-photolysis time-resolved microwave conductivity (FP-TRMC) under $\mathrm{N}_{2}$ atmosphere. In the measurement, charge carriers are generated upon photo excitation and the local motion of the carriers can be probed via dielectric loss of the low-power microwave probes. The observable region of charge carriers can be extended to a very short time region, which allows us to obtain the intrinsic chargecarrier mobility within several nanometers after laser pulse irradiation under a rapidly oscillating electric field ${ }^{44,45}$. This technique is devoid of grain boundaries, impurities or crystal size, which reflects long-range $(>10 \mathrm{~nm})$ carrier transport along the ordered structure in crystalline domains ${ }^{46}$. Thus, the FP-TRMC photoconductivity $(\phi \Sigma \mu)$, where $\phi$ and $\Sigma \mu$ represent the photocarrier generation yield and the sum of the photogenerated charge carrier mobilities, respectively ${ }^{46}$, cannot be affected by the length of the crystals, but gives an estimate of the actual crystallinity in nanometer-scale level (see Supplementary Note 2). After $5 \mathrm{~min}$ growth in $\mathrm{sc}-\mathrm{CO}_{2}$, the photoconductivity transients of sc- $\mathrm{COF}_{\text {TP-Py }}$ show a rapid rise within the time constant of the present set of apparatus in current, with a maximum $\phi \Sigma \mu$ value of $5.8 \times 10^{-6} \mathrm{~cm}^{2} \mathrm{~V}^{-1} \mathrm{~s}^{-1}$ at an excitation photon density of $1.8 \times 10^{16}$ photons $\mathrm{cm}^{-2} . \phi \Sigma \mu$ reaches $\sim 6.8 \times 10^{-6} \mathrm{~cm}^{2} \mathrm{~V}^{-1} \mathrm{~s}^{-1}$ after growth in $\mathrm{sc}-\mathrm{CO}_{2}$ for $20 \mathrm{~min}$ to $12 \mathrm{~h}$ (Fig. 6). As a comparison, $\phi \Sigma \mu$ of os- $\mathrm{COF}_{\mathrm{TP}-\mathrm{Py}}$ cannot reach the same value, even after growth for 3 days. Therefore, ultra-fast polymerization in sc- $\mathrm{CO}_{2}$ not only obtains large-sized single crystals, but also results in comparable or higher crystal quality compared with os-COFs grown for days.

\section{Discussion}

Practical application of COFs received limited success due to challenges in synthesizing high-quality products rapidly, industrycompatibly and environmentally friendly. Supercritically solvothermal polymerization reduces the time needed for growing large-sized 2D COF single crystals from months to minutes, which is hard or even impossible previously. Despite ultra-fast growth rate, it increases the crystal size up to $0.2 \mathrm{~mm}$ (sc-COF
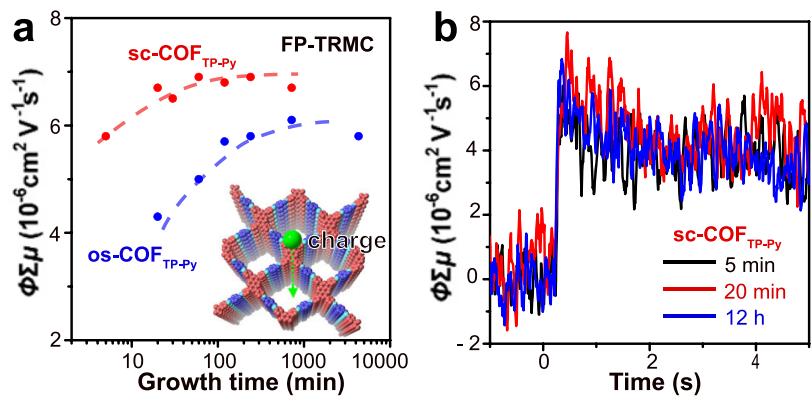

Fig. 6 FP-TRMC measurement. a Photoconductivity $(\phi \Sigma \mu)$ of sc-COF $\mathrm{TP}_{\mathrm{TPy}}$ and os-COF $\mathrm{FP}_{\mathrm{TP}}$ as a function of the growth time. $\mathbf{b}$ FP-TRMC transients of the sc-COF $\mathrm{TP}_{\mathrm{T} \text { - }}$ grown for $5 \mathrm{~min}, 20 \mathrm{~min}$ and $12 \mathrm{~h}$, respectively.

larger than the largest single crystals of $2 \mathrm{D}$ COFs reported previously ${ }^{8}$, and also improves the quality and photoconductivity performance. Sc-COFs can be further purified and activated in sc$\mathrm{CO}_{2}$ (Supplementary Fig. 28). Although the solubility of sc- $\mathrm{CO}_{2}$ isn't so strong as organic solvents, which results in a few of uncleaned monomers or oligomers in the nanochannels of $2 \mathrm{D}$ COFs, it is impressive that the surface area still reaches $1710 \mathrm{~m}^{2} \mathrm{~g}^{-1}$, comparable to that of os-COF $\mathrm{COP}_{\mathrm{TPy}}\left(1947 \mathrm{~m}^{2} \mathrm{~g}^{-1}\right)$. Thus, the entire procedure is environmentally friendly without using amounts of organic solvents. Considering these as well as wide usage of sc- $\mathrm{CO}_{2}$ in industry, it solves all the above challenges with universality for different linkages of $2 \mathrm{D} \mathrm{COFs}$, which greatly shortens the gap between synthesis and practical applications. Moreover, obstacle in synthesis hampers researches and applications of $2 \mathrm{D}$ COF single crystals. This work solves the problem and finds that the high quality and singlecrystalline nature of sc-COFs give rise to highly polarized PL, showing that the $2 \mathrm{D}$ COF single crystals produced in sc- $\mathrm{CO}_{2}$ have great potential in special application fields which previous polycrystalline samples haven't. It is worth noting that there is a general belief that slow crystallization is necessary to grow high-quality single crystals $^{1}$. In contrary, this result indicates ultra-fast polymerization in sc- $-\mathrm{CO}_{2}$ can produce single crystals with both larger size and higher quality, compared with slow crystallization, overcoming the contradiction between growth time and product quality. This work provides a new understanding of ultra-fast single-crystal polymerization in supercritical liquid. Besides boronate ester and imine 2D COFs, it also holds great promise in the efficient and precise construction of various COFs and other covalent crystalline materials, opening up other opportunities for not only fundamental research but also practical application of these materials and their single crystals.

\section{Methods}

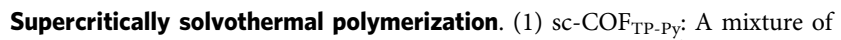
terephthalaldehyde (TP, $5.4 \mathrm{mg}, 0.04 \mathrm{mmol})$ and 1,3,6,8-tetra( $4^{\prime}$-aminophenyl) pyrene (Py, $11.3 \mathrm{mg}, 0.02 \mathrm{mmol})$ was dissolved in $100 \mu \mathrm{L}$-butyl alcohol $(0.25 \%$ vol., as a cosolvent) $(\mathrm{n}-\mathrm{BuOH})$ and $100 \mu \mathrm{L}$ acetic acid, then transferred to a $40 \mathrm{~mL}$ stainless steel reactor. The system was charged with $8 \mathrm{MPaCO}_{2}$, heated to $80^{\circ} \mathrm{C}$, and reacted for $5 \mathrm{~min}$. After the reaction, the reactor was slowly depressurized at a rate of 1-2 $\mathrm{MPa} \mathrm{min}^{-1}$. The precipitate was collected by filtration, washed with acetone, tetrahydrofuran, and dried in a vacuum oven at $100{ }^{\circ} \mathrm{C}$.

(2) $\mathrm{sc}^{-\mathrm{COF}_{\mathrm{TB}} \mathrm{BA}}$ : A mixture of 1,3,5-triformylbenzene (TB, $9.7 \mathrm{mg}, 0.06 \mathrm{mmol}$ ) and 4,4'-biphenyldiamine (BA, $16.6 \mathrm{mg}, 0.09 \mathrm{mmol}$ ) was dissolved in $100 \mu \mathrm{L}$ $\mathrm{n}-\mathrm{BuOH}$ and $100 \mu \mathrm{L}$ acetic acid, and transferred to a $40 \mathrm{~mL}$ stainless steel reactor. The system was charged with $8 \mathrm{MPa} \mathrm{CO}_{2}$, heated to $80^{\circ} \mathrm{C}$, and reacted for $5 \mathrm{~min}$ After the reaction, the reactor was slowly depressurized at a rate of 1-2 $\mathrm{MPa} \mathrm{min}{ }^{-1}$ The precipitate was collected by filtration, washed with acetone, tetrahydrofuran, and dried in vacuum oven at $100^{\circ} \mathrm{C}$.

(3) sc- $\mathrm{COF}_{5}$ : A mixture of 2,3,6,7,10,11-hexahydroxytriphenylene (HHTP) (11 $\mathrm{mg}, 0.034 \mathrm{mmol})$ and benzene diboronic acid (BDBA) $(8.28 \mathrm{mg}, 0.05 \mathrm{mmol})$ was dissolved in $200 \mu \mathrm{L} \mathrm{n}-\mathrm{BuOH}$, and transferred to a $40 \mathrm{~mL}$ stainless steel reactor and the system was heated to $90^{\circ} \mathrm{C}$ and pressurized up to $9 \mathrm{MPa}^{\mathrm{CO}} \mathrm{O}_{2}$, reacted for $90 \mathrm{~min}$. After the reaction, the reactor was slowly depressurized at a rate of 1-2 MPa $\min ^{-1}$. The precipitate was collected by filtration, washed with acetone, and dried in vacuum overnight. 
Solvothermal polymerization. (1) os- $\mathrm{COF}_{\mathrm{TP}-\mathrm{Py}}$ : A mixture of terephthalaldehyde (TP, $5.4 \mathrm{mg}, 0.04 \mathrm{mmol}$ ) and 1,3,6,8-tetra $\left(4^{\prime}\right.$-aminophenyl) pyrene (Py, $11.3 \mathrm{mg}$, $0.02 \mathrm{mmol}$ ) was dissolved in $0.5 \mathrm{~mL} \mathrm{n-BuOH}$ and $0.5 \mathrm{~mL} 1,2$-dichlorobenzene (o$\mathrm{DCB})$, and transferred to a reaction tube. After sonication for $5 \mathrm{~min}$ at room temperature, the mixture was added $0.1 \mathrm{~mL} 6 \mathrm{M}$ aqueous acetic acid. Then the tube was degassed through three freeze-pump-thaw cycles, sealed under vacuum and heated at $120^{\circ} \mathrm{C}$ for 3 days. After the reaction, the precipitate was collected by filtration, washed with acetone, tetrahydrofuran, and dried in vacuum oven at $100^{\circ} \mathrm{C}^{47}$.

(2) os-COF $\mathrm{COF}_{\mathrm{TB}-\mathrm{BA}}$ : A mixture of 1,3,5-triformylbenzene (TB, $9.7 \mathrm{mg}, 0.06 \mathrm{mmol}$ ) and 4,4'-biphenyldiamine (BA, $16.6 \mathrm{mg}, 0.09 \mathrm{mmol}$ ) was dissolved in $0.5 \mathrm{~mL}$ mesitylene and $0.5 \mathrm{~mL}$ dioxane, and transferred to a reaction tube. After sonication for $5 \mathrm{~min}$ at room temperature, the mixture was added $0.1 \mathrm{~mL} 6 \mathrm{M}$ aqueous acetic acid. Then, the tube was degassed through three freeze-pump-thaw cycles, sealed under vacuum and heated at $120^{\circ} \mathrm{C}$ for 3 days. After reaction, the precipitate was collected by filtration, washed by acetone, tetrahydrofuran and dried in vacuum oven at $100^{\circ} \mathrm{C}^{27}$.

(3) os- $\mathrm{COF}_{5}$ : A mixture of 2,3,6,7,10,11-hexahydroxytriphenylene (HHTP) (11 $\mathrm{mg}, 0.034 \mathrm{mmol})$ and benzene diboronic acid (BDBA) $(8.28 \mathrm{mg}, 0.05 \mathrm{mmol})$ was dissolved in $0.5 \mathrm{~mL}$ mesitylene and $0.5 \mathrm{~mL}$ dioxane, and transferred to a reaction tube. After sonication for $5 \mathrm{~min}$ at room temperature. Then the tube was degassed through three freeze-pump-thaw cycles, sealed under vacuum, and heated at $100^{\circ} \mathrm{C}$ for 3 days. After the reaction, the precipitate was collected by filtration, washed with acetone and dried in vacuum overnight ${ }^{48}$.

Characterization. The samples were measured by PXRD (PXRD, Bruker, D8). All the COFs were recorded in the $2 \theta$ range between $2^{\circ}$ and $40^{\circ}$. The radiation was $\mathrm{Cu}$ $\mathrm{Ka}(\lambda=1.54 \AA)$, and the data collection was carried out using an Aluminum holder at a scan speed of $1^{\circ} \mathrm{min}^{-1}$ and a step size of $0.02^{\circ}$. Fourier transform infrared (FTIR) spectra were collected using a thermofisher Nicolet 6700 spectrometer.

TEM images were collected using FEI Tecnai G2 F20 S-Twin (acceleration voltage: $200 \mathrm{kV}$ ). SEM images were collected using Zeiss Gemini SEM500. The SEM samples were prepared by evaporating a drop of alcohol with COFs on a clean $\mathrm{Si} / \mathrm{SiO}_{2}$ wafer. OM and cross-polarized $\mathrm{OM}$ images were collected using a polarized OM (Leica DM2500P).

The $\mathrm{N}_{2}$ adsorption-desorption experiments were conducted on an Autosorb $\mathrm{iQ}_{2}$ (Anton Paar) surface area analyser. The os- $\mathrm{COF}_{\mathrm{TP}-\mathrm{Py}}$ sample was washed in THF by Soxhlet's extraction for 3 days, dried in vacuum for $12 \mathrm{~h}$, and degassed at $120^{\circ} \mathrm{C}$ for $12 \mathrm{~h}$ before the measurement. The sc-COF ${ }_{\mathrm{TP}-\mathrm{Py}}$ sample was purified by liquid $\mathrm{CO}_{2}$ for 5 times, kept in sc- $\mathrm{CO}_{2}\left(40^{\circ} \mathrm{C} 8 \mathrm{MPa}\right)$ for one hour (Samdri ${ }^{\oplus}-\mathrm{PVT}-3 \mathrm{D}$, Tousimis) and degassed at $0.13 \mathrm{MPa} \mathrm{min}^{-1}$ before the measurements ${ }^{49} . \mathrm{N}_{2}$ isotherms were recorded at $77 \mathrm{~K}$ by using ultra-high purity N2 (99.999\% purity). The surface area was determined using Brunauer-Emmett-Teller (BET) adsorption model.

Growth and characterization of 3D-ordered sc-COFs. After being treated with aqueous hydrogen peroxide and concentrated sulfuric acid (vol./vol. = 3:1), $\mathrm{Si} / \mathrm{SiO}_{2}$ wafer was cleaned with isopropanol, acetone, and DI water, respectively. Then, the wafer was blown dry by a $\mathrm{N}_{2}$ gun and suspended vertically in $10 \%$ wt. aqueous polystyrene (PS) microspheres dispersion (Thermo Corp.). The dispersion was placed in an oven at $40^{\circ} \mathrm{C}$ overnight. By controlling the evaporation rate, highly ordered 3D monoliths of PS were obtained on the $\mathrm{SiO}_{2} / \mathrm{Si}_{\text {wafer }}{ }^{50}$.

To grow the sc-COF $\mathrm{TB}_{\mathrm{TB}} \mathrm{BA}$ in the highly ordered 3D monolith of PS, $16 \mathrm{mg} \mathrm{1,3,5-}$ benzenetricarboxaldehyde and $27.6 \mathrm{mg} 4,4^{\prime}$-diaminobiphenyl were mixed within $0.1 \mathrm{~mL}$ $\mathrm{n}-\mathrm{BuOH}$ and $0.1 \mathrm{~mL}$ acetic acid, transferred into stainless steel reactor, then a piece of PS colloid crystal was immerged into the solution. Then the reactor was heated to $80^{\circ} \mathrm{C}$ and charged with $8 \mathrm{MPa} \mathrm{CO}_{2}$. After a reaction for $12 \mathrm{~h}$, the reactor was cooled to room temperature and slowly depressurized at a rate of $1-2 \mathrm{MPa} \mathrm{min}^{-1}$. The wafer was washed with THF and acetone, and characterized by SEM.

Angle-dependent photoluminescence measurement. The angle-dependent photoluminescence $(\mathrm{PL})$ spectra were measured by a Horiba iHR550 spectrometer equipped with a silicon CCD detector array ${ }^{51}$. A $532 \mathrm{~nm}$ laser (spot size $\sim 1 \mu \mathrm{m}$, laser power of $70 \mu \mathrm{W}$ ) was used as the excitation source. The incident laser was focused by a Nikon Eclipse Ti microscope with a $50 \times(\mathrm{NA}=0.7)$ objective. In the measurements, the polarization direction of the incident laser light was parallel to the long axis of COF crystals. A linear polarizer was placed in the collecting optical path to detect the angle dependence of the PL emission. The polarized PL images were collected using Olympus BX-51-P fluorescence microscope at different polarization angles (by rotating the stage). We used a filter set which provided a narrow wavelength range ( 450 to $490 \mathrm{~nm}$ ) for excitation, and allowed that emitted light at wavelengths above $515 \mathrm{~nm}$ passed to the detector. All optical experiments were performed in ambient environment and at room temperature.

Flash photolysis time-resolved microwave conductivity test. Flash photolysis time-resolved microwave conductivity (FP-TRMC) was performed by in situ TRMC system ${ }^{52}$. A high degree of sensitivity in the conductivity measurement was realized in a resonant cavity. The resonant frequency and power of microwave probes were set at $\sim 9.1 \mathrm{GHz}$ and $3 \mathrm{~mW}$, respectively. Charge carriers were generated upon direct excitation of solid samples with third-harmonic generation $(\lambda=355 \mathrm{~nm})$ from a Spectra-Physics model INDI-HG Nd: YAG laser (Continuum Inc., Surelite II, 5-8 ns pulse duration, $10 \mathrm{~Hz}$ ), and the conductivity transients were recorded by a digital oscilloscope (Tektronix, TDS 3032B). All the experiments were performed at $25^{\circ} \mathrm{C}$ in nitrogen.

\section{Data availability}

All data supporting the findings of this work are available within this paper and its Supplementary Information (figures, videos). Other data are available from the corresponding author upon request.

Received: 18 August 2020; Accepted: 14 June 2021; Published online: 23 August 2021

\section{References}

1. Pamplin, B. R. Crystal Growth (Pergamon Press, 1980).

2. Rowan, S. J., Cantrill, S. J., Cousins, G. R. L., Sanders, J. K. M. \& Stoddart, J. F. Dynamic covalent chemistry. Angew. Chem. Int. Ed. 41, 898-952 (2002).

3. Waller, P. J., Gándara, F. \& Yaghi, O. M. Chemistry of covalent organic frameworks. Acc. Chem. Res. 48, 3053-3063 (2015).

4. Wang, W. \& Schlüter, A. D. Synthesis of 2D polymers: a critical perspective and a look into the future. Macromol. Rapid Commun. 40, 1800719 (2019).

5. Smith, B. J. \& Dichtel, W. R. Mechanistic studies of two-dimensional covalent organic frameworks rapidly polymerized from initially homogenous conditions. J. Am. Chem. Soc. 136, 8783-8789 (2014).

6. Li, H. et al. Nucleation and growth of covalent organic frameworks from solution: the example of COF-5. J. Am. Chem. Soc. 139, 16310-16318 (2017).

7. Navarro, J. A. R. The dynamic art of growing COF crystals. Science 361, 35 (2018).

8. Evans, A. M. et al. Seeded growth of single-crystal two-dimensional covalent organic frameworks. Science 361, 52-57 (2018).

9. Ma, T. et al. Single-crystal X-ray diffraction structures of covalent organic frameworks. Science 361, 48-52 (2018).

10. Ding, S.-Y. \& Wang, W. Covalent organic frameworks (COFs): from design to applications. Chem. Soc. Rev. 42, 548-568 (2013).

11. Segura, J. L., Mancheno, M. J. \& Zamora, F. Covalent organic frameworks based on Schiff-base chemistry: synthesis, properties and potential applications. Chem. Soc. Rev. 45, 5635-5671 (2016).

12. Smith, B. J., Overholts, A. C., Hwang, N. \& Dichtel, W. R. Insight into the crystallization of amorphous imine-linked polymer networks to $2 \mathrm{D}$ covalent organic frameworks. Chem. Commun. 52, 3690-3693 (2016).

13. Koo, B. T., Heden, R. F. \& Clancy, P. Nucleation and growth of $2 \mathrm{D}$ covalen organic frameworks: polymerization and crystallization of COF monomers. Phys. Chem. Chem. Phys. 19, 9745-9754 (2017).

14. Feng, X., Ding, X. \& Jiang, D. Covalent organic frameworks. Chem. Soc. Rev. 41, 6010-6022 (2012)

15. Cote, A. P. et al. Porous, crystalline, covalent organic frameworks. Science $\mathbf{3 1 0}$ 1166-1170 (2005).

16. El-Kaderi, H. M. et al. Designed synthesis of 3D covalent organic frameworks. Science 316, 268-272 (2007)

17. Ding, S.-Y. et al. Construction of covalent organic framework for catalysis: $\mathrm{Pd} /$ COF-LZU1 in Suzuki-Miyaura coupling reaction. J. Am. Chem. Soc. 133, 19816-19822 (2011)

18. Wang, X. et al. Sulfone-containing covalent organic frameworks for photocatalytic hydrogen evolution from water. Nat. Chem. 10, 1180-1189 (2018).

19. Wan, S., Guo, J., Kim, J., Ihee, H. \& Jiang, D. A photoconductive covalent organic framework: self-condensed arene cubes composed of eclipsed 2D polypyrene sheets for photocurrent generation. Angew. Chem. Int. Ed. $\mathbf{4 8}$, 5439-5442 (2009)

20. Li, X. et al. Tuneable near white-emissive two-dimensional covalent organic frameworks. Nat. Commun. 9, 2335 (2018).

21. Liu, Y. et al. Weaving of organic threads into a crystalline covalent organic framework. Science 351, 365-369 (2016).

22. Kandambeth, S. et al. Construction of crystalline $2 \mathrm{D}$ covalent organic frameworks with remarkable chemical (acid/base) stability via a combined reversible and irreversible route. J. Am. Chem. Soc. 134, 19524-19527 (2012).

23. Karak, S. et al. Constructing ultraporous covalent organic frameworks in seconds via an organic terracotta process. J. Am. Chem. Soc. 139, 1856-1862 (2017).

24. Campbell, N. L., Clowes, R., Ritchie, L. K. \& Cooper, A. I. Rapid microwave synthesis and purification of porous covalent organic frameworks. Chem. Mater. 21, 204-206 (2009). 
25. Sun, J.-K., Sobolev, Y. I., Zhang, W., Zhuang, Q. \& Grzybowski, B. A. Enhancing crystal growth using polyelectrolyte solutions and shear flow. Nature 579, 73-79 (2020).

26. Spitler, E. L. \& Dichtel, W. R. Lewis acid-catalysed formation of twodimensional phthalocyanine covalent organic frameworks. Nat. Chem. 2, 672-677 (2010).

27. Bai, L., Gao, Q. \& Zhao, Y. Two fully conjugated covalent organic frameworks as anode materials for lithium ion batteries. J. Mater. Chem. A. 4, 14106-14110 (2016).

28. Ascherl, L. et al. Solvatochromic covalent organic frameworks. Nat. Commun. 9, 3802 (2018)

29. Sang, X. et al. Ionic liquid accelerates the crystallization of Zr-based metal-organic frameworks. Nat. Commun. 8, 175 (2017).

30. Knez, Ž. et al. Industrial applications of supercritical fluids: a review. Energy 77, 235-243 (2014).

31. Kankala, R. K., Zhang, Y. S., Wang, S.-B., Lee, C.-H. \& Chen, A.-Z. Supercritical fluid technology: an emphasis on drug delivery and related biomedical applications. Adv. Health Mater. 6, 1700433 (2017).

32. Byrappa, K., Ohara, S. \& Adschiri, T. Nanoparticles synthesis using supercritical fluid technology - towards biomedical applications. Adv. Drug. Deliv. Rev. 60, 299-327 (2008).

33. Johnston, K. P. Making nanoscale materials with supercritical fluids. Science 303, 482-483 (2004).

34. Caballero, Ana et al. Silver-catalyzed C-C bond formation between methane and ethyl diazoacetate in supercritical $\mathrm{CO}_{2}$. Science 332, 835-838 (2011)

35. Ingrosso, F. \& Ruiz-Lopez, M. F. Modeling solvation in supercritical $\mathrm{CO}_{2}$. Chemphyschem 18, 2560-2572 (2017).

36. Wakayama, H. \& Fukushima, Y. Supercritical $\mathrm{CO}_{2}$ as a solvent for synthesis of nanoporous materials. Ind. Eng. Chem. Res. 39, 4641-4645 (2000).

37. Barim, S. B., Uzunlar, E., Bozbag, S. E. \& Erkey, C. Supercritical deposition: a powerful technique for synthesis of functional materials for electrochemical energy conversion and storage. J. Electrochem. Soc. 167, 054510 (2020).

38. Desimone, J. M. et al. Dispersion polymerizations in supercritical carbon dioxide. Science 265, 356-359 (1994).

39. Sun, M. J. et al. Photoluminescent anisotropy amplification in polymorphic organic nanocrystals by light-harvesting energy transfer. J. Am. Chem. Soc. 141, 6157-6161 (2019).

40. Motamen, S. et al. Revealing order and disorder in films and single crystals of a thiophene-based oligomer by optical spectroscopy. ACS Photonics 3, 2315-2323 (2016).

41. Wang, J., Gudiksen, M. S., Duan, X. F., Cui, Y. \& Lieber, C. M. Highly polarized photoluminescence and photodetection from single indium phosphide nanowires. Science 293, 1455-1457 (2001).

42. Saeki, A., Ohsaki, S.-i, Seki, S. \& Tagawa, S. Electrodeless determination of charge carrier mobility in poly(3-hexylthiophene) films incorporating perylenediimide as photoconductivity sensitizer and spectroscopic probe. $J$. Phys. Chem. C 112, 16643-16650 (2008).

43. Wan, S. et al. Covalent organic frameworks with high charge carrier mobility. Chem. Mater. 23, 4094-4097 (2011).

44. Dicker, G., de Haas, M. P., Siebbeles, L. D. A. \& Warman, J. M. Electrodeless time-resolved microwave conductivity study of charge-carrier photogeneration in regioregular poly(3-hexylthiophene) thin films. Phys. Rev. $B$ 70, 045203 (2004).

45. Feng, X. et al. High-rate charge-carrier transport in porphyrin covalent organic frameworks: switching from hole to electron to ambipolar conduction. Angew. Chem. Int. Ed. 124, 2672-2676 (2012).

46. Saeki, A., Koizumi, Y., Aida, T. \& Seki, S. Comprehensive approach to intrinsic charge carrier mobility in conjugated organic molecules, macromolecules, and supramolecular architectures. Acc. Chem. Res. 45, 1193-1202 (2012).

47. Meng, Y., Lin, G., Ding, H., Liao, H. \& Wang, C. Impregnation of sulfur into a 2D pyrene-based covalent organic framework for high-rate lithium-sulfur batteries. J. Mater. Chem. A 6, 17186-17191 (2018).

48. Guo, Z. et al. Fast ion transport pathway provided by polyethylene glycol confined in covalent organic frameworks. J. Am. Chem. Soc. 141, 1923-1927 (2019).
49. Vitaku, E. \& Dichtel, W. R. Synthesis of 2D imine-linked covalent organic frameworks through formal transimination reactions. J. Am. Chem. Soc. 139, 12911-12914 (2017).

50. Huang, Y. et al. Colloidal photonic crystals with narrow stopbands assembled from low-adhesive superhydrophobic substrates. J. Am. Chem. Soc. 134, 17053-17058 (2012).

51. Tauber, D., Dobrovolsky, A., Camacho, R. \& Scheblykin, I. G. Exploring the electronic band structure of organometal halide perovskite via photoluminescence anisotropy of individual nanocrystals. Nano Lett. 16, 5087-5094 (2016).

52. Ding, X. et al. An n-channel two-dimensional covalent organic framework. J. Am. Chem. Soc. 133, 14510-14513 (2011).

\section{Acknowledgements}

We thank Songhai Xie, Xiali Zhang, Qingsong Wu from Fudan University for their aid in the TEM test. This work was supported by the National Key R\&D Program of China (2021YFE0201400, 2018YFA0703200), National Natural Science Foundation of China (51773041, 61890940, 21603038), Shanghai Committee of Science and Technology in China (18ZR1404900), the Strategic Priority Research Program of the Chinese Academy of Sciences (XDB30000000) and Fudan University.

\section{Author contributions}

D.W. obtained ideas, designed research, and supervised the project. L.P., Q.G., D.H. and L.Z. prepared the samples. L.P. and L.S. produced 3D-ordered structures of COFs. L.P. and L.W. did SEM. L.P. and D.W. did TEM, POM, FT-IR, and XRD. H.X. and Q.L. measured BET. L.P., C.S. and H.Y. measured angle-dependent PL. S.G., T. S. and S.S. measured FP-TRMC. D.W., L.P. and Y.L. prepared the manuscript. All authors commented on the manuscript.

\section{Competing interests}

The authors declare no competing interests.

\section{Additional information}

Supplementary information The online version contains supplementary material available at https://doi.org/10.1038/s41467-021-24842-x.

Correspondence and requests for materials should be addressed to D.W.

Peer review information Nature Communications thanks Rahul Banerjee and the other anonymous, reviewer(s) for their contribution to the peer review of this work. Peer reviewer reports are available.

Reprints and permission information is available at http://www.nature.com/reprints

Publisher's note Springer Nature remains neutral with regard to jurisdictional claims in published maps and institutional affiliations.

Open Access This article is licensed under a Creative Commons Attribution 4.0 International License, which permits use, sharing, adaptation, distribution and reproduction in any medium or format, as long as you give appropriate credit to the original author(s) and the source, provide a link to the Creative Commons license, and indicate if changes were made. The images or other third party material in this article are included in the article's Creative Commons license, unless indicated otherwise in a credit line to the material. If material is not included in the article's Creative Commons license and your intended use is not permitted by statutory regulation or exceeds the permitted use, you will need to obtain permission directly from the copyright holder. To view a copy of this license, visit http://creativecommons.org/ licenses/by/4.0/

(C) The Author(s) 2021 